\title{
A massive natural disaster, the Great East Japan Earthquake, and the incidence of dialysis due to end-stage kidney disease
}

\author{
Michiaki Abe ${ }^{1,2,3}$ (1) $\cdot$ Tetsuya Akaishi $^{2} \cdot$ Koto Ishizawa $^{1,2} \cdot$ Hirohisa Shinano $^{4} \cdot$ Hiroshi Ohtomo $^{4} \cdot$ Kazuhiko Orikasa $^{4}$. \\ Shin Takayama ${ }^{1} \cdot$ Atsuko Masaura $^{1} \cdot$ Mariko Miyazaki $^{3} \cdot$ Takaaki Abe $^{3} \cdot$ Kenichi Yokota $^{4} \cdot$ Tadashi Ishii $^{1,2}$
}

Received: 13 April 2021 / Accepted: 6 August 2021 / Published online: 12 October 2021

(C) Italian Society of Nephrology 2021

\begin{abstract}
Background Disaster-related stress can increase blood pressure and the incidence of cardiovascular diseases. However, the role of massive disasters in the development of end-stage kidney disease (ESKD) remains unknown. We investigated the incidence and different causes of dialysis initiation in patients with chronic kidney disease in a city affected by the Great East Japan Earthquake.

Methods This was a single-center, retrospective observational study. All patients who initiated or were treated with dialysis at Kesennuma City Hospital between 2007 and 2020 were enrolled. The year of dialysis initiation was retrospectively determined based on the initiation date. The causative renal diseases that led to the need for dialysis initiation were divided into four groups: diabetic nephropathy, hypertensive renal disease, glomerulonephritis, and others.

Results Age at dialysis initiation differed significantly among the four groups $(p=0.0262)$. There was a significant difference in the numbers of the four groups before and after the Great East Japan Earthquake $(p=0.0193)$. The age of hypertensive renal disease patients was significantly higher than those of patients with diabetic nephropathy $(p=0.0070)$ and glomerulonephritis $(p=0.0386)$ after the disaster. The increasing number of dialysis initiations after the Great East Japan Earthquake appeared to be associated with changes in hypertensive renal diseases; the number peaked after 10 years.

Conclusions There was an increase in the number of dialysis initiations, especially caused by hypertensive renal diseases, for up to 10 years after the Great East Japan Earthquake.
\end{abstract}

Michiaki Abe

michiabe@med.tohoku.ac.jp

1 Department of Education and Support for Regional Medicine, Tohoku University Hospital, Sendai, Japan

2 Tohoku Medical Megabank Organization, Tohoku University, Sendai, Japan

3 Division of Nephrology, Endocrinology and Vascular Medicine, Tohoku University Graduate School of Medicine, Sendai, Japan

4 Kesennuma City Hospital, Kesennuma, Japan 


\section{Graphic abstract}
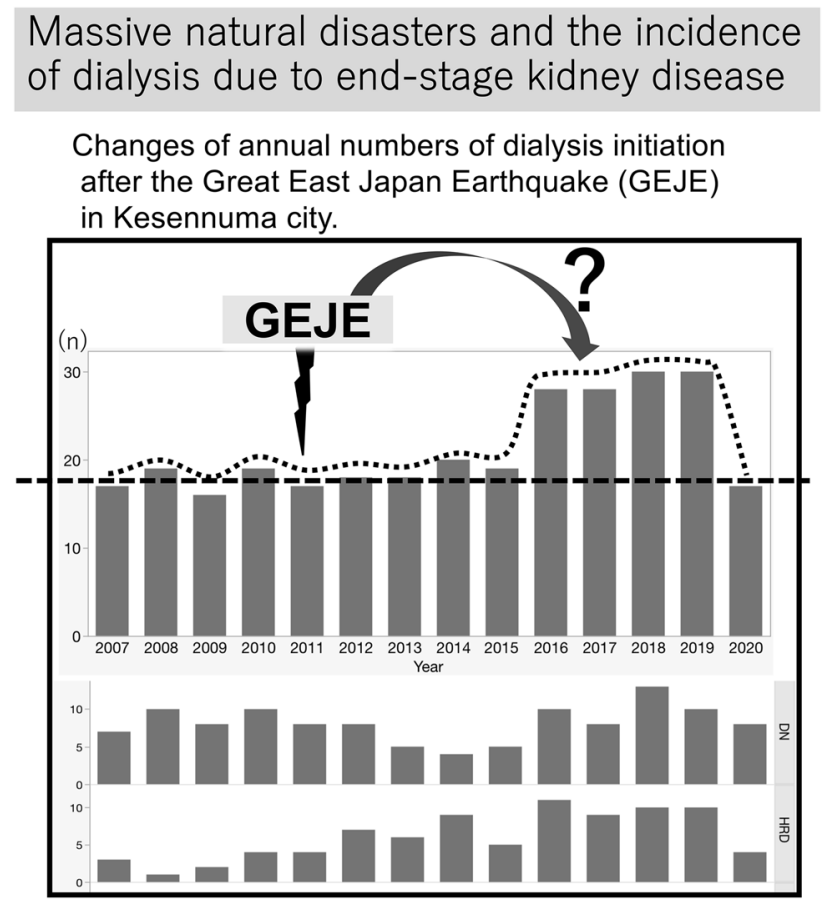

Keywords Massive natural disaster $\cdot$ Dialysis initiation $\cdot$ End-stage kidney disease $\cdot$ Hypertensive renal disease $\cdot$ Diabetic nephropathy

\section{Introduction}

Hypertension is a risk factor for chronic kidney disease; furthermore, renal dysfunction also leads to high blood pressure.

Extremely stressful experiences, such as those caused by natural disasters, are commonly known as allostatic loads.
The psychological load may disrupt blood pressure and glycemic control [1, 2]. Consequently, poor blood pressure and glycemic control have been reported to increase the risk of cardiovascular events (e.g., heart failure, ischemic heart disease) and stroke in the short term [2-4]. However, the long-term effects of a massive natural disaster are less known and their effects on the development and prognosis of
Table 1 Causal renal diseases for dialysis initiation

\begin{tabular}{lclllll}
\hline & Total & DN & HRD & GN & Others & $p$ value \\
\hline $\mathrm{N}$ & 296 & 114 & 85 & 54 & 43 & \\
Gender [female, $\mathrm{n}$ ] & 91 & 33 & 31 & 16 & 11 & $0.5816^{*}$ \\
Age [years] & & & & & & \\
$\quad$ Median & 71 & 70 & 76 & 71 & 71 & $0.0262^{* *}$ \\
1st quartile & 63 & 62.75 & 65 & 60.75 & 62 & \\
3rd quartile & 78 & 77 & 79.5 & 76.25 & 81 & \\
Number of patients requiring dialysis initiation before and after the earthquake & & \\
Before GEJE & 81 & 39 & 13 & 17 & 12 & $0.0193^{*}$ \\
After GEJE & 215 & 75 & 72 & 37 & 31 & \\
\hline
\end{tabular}

GEJE the Great East Japan Earthquake, GN glomerulonephritis, $D N$ diabetic nephropathy, HRD hypertensive renal disease

* $p$ value by Fisher's exact test

** $p$ value by Wilcoxon test 
renal diseases have not been elucidated. This study focused on the increase in dialysis initiation after a massive natural disaster, the Great East Japan Earthquake, to evaluate the effects of such disasters on the risk of end-stage kidney disease (ESKD).

In March 2011, Japan was hit by the Great East Japan Earthquake.

This earthquake scored 9.0 on the Richter scale, and was followed by a devastating tsunami along the coastal areas. The disaster caused more than 15,000 casualties, while more than 2500 people have been reported missing as of May 2020. Many cities and towns in the coastal areas, including Kesennuma City (Miyagi, Japan), were almost completely destroyed by the tsunami. Following the Great East Japan Earthquake, Kesennuma City was isolated; Kesennuma City Hospital is the only medical facility in the city with nephrology and dialysis facilities. It is noteworthy that despite a decrease in the population, there was an increase in dialysis initiation several years after the Great East Japan Earthquake [5]. To evaluate the causes of this observation, we studied the causative renal diseases that necessitated dialysis initiation before and after the Great East Japan Earthquake in our remote coastal city.

\section{Materials and methods}

\section{Patient enrollment}

Patients on dialysis initiation in Kesennuma City Hospital were enrolled, as were patients for whom dialysis was initiated in other facilities and returned to Kesennuma for maintenance dialysis.
Exclusion criteria were: dialysis initiation before 2006 and the inability to shift to chronic outpatient dialysis.

All enrolled patients living in Kesennuma City or neighboring towns were victims of the Great East Japan Earthquake, and started dialysis between January 2007 and December 2020. According to the guidelines of the Japanese Society of Dialysis, the following indications to initiate dialysis are retained: serum Creatinine $\geq 8 \mathrm{mg} / \mathrm{dL}$, creatinine clearance $(\mathrm{Ccr}) \leq 10 \mathrm{~mL} / \mathrm{min}$, difficulty in daily life activities, uncontrollable uremic symptoms. The study was approved by the Ethics Committee of Kesennuma City Hospital (IRB No. 1067).

\section{Definition of renal disease groups}

The renal diseases that led to the need for dialysis initiation were classified into one of the following four groups: diabetic nephropathy (DN); glomerulonephritis (GN); hypertensive renal disease (HRD), including arteriolosclerosis and arteriosclerotic renal ischemia; and others. Diabetes mellitus, maintained kidney size and high urinary albumin excretion, or renal biopsies were used for diagnosis of DN [6].

Patients with HRD had symptoms of chronic hypertension and low-grade proteinuria $(<1.0 \mathrm{~g} / \mathrm{gCr})$ during the mild and moderate CKD phases, usually with atrophic kidneys and without pathological urinary casts. The "other" group included: autosomal dominant polycystic kidney disease, interstitial nephritis, and other unknown causes.
Table 2 Comparisons of gender and age of the causal renal diseases before and after GEJE

\begin{tabular}{lllllll}
\hline & Total & DN & HRD & GN & Others & $p$ value \\
\hline Gender [female; $\mathrm{n}$ ] & & & & & & \\
Before GEJE & 26 & 15 & 2 & 7 & 2 & $0.2645^{*}$ \\
After GEJE & 65 & 18 & 29 & 9 & 9 & $0.1531^{*}$ \\
Age [years] & & & & & & \\
Before GEJE & & $70 * *$ & $68^{* *}$ & $70^{* *}$ & 76 & $0.7430^{* *}$ \\
Median & 70 & $60^{* *}$ & $57^{* *}$ & $58^{* *}$ & 62 & \\
1st quartile & 59.5 & $76^{* *}$ & $77.5^{* *}$ & $76.5^{* *}$ & 78 & \\
3rd quartile & 77 & & & & & \\
After GEJE & & $71^{* *}$ & $76^{* *}$ & $71^{* *}$ & 69 & $0.0480^{* *}$ \\
Median & 72 & $64^{* *}$ & $68^{* *}$ & $61.5^{* *}$ & 62 & \\
1st quartile & 64 & $77^{* *}$ & $80^{* *}$ & $76.5^{* *}$ & 82 & \\
3rd quartile & 78 & &
\end{tabular}

GEJE the Great East Japan Earthquake, GN glomerulonephritis, $D N$ diabetic nephropathy, $H R D$ hypertensive renal disease

* $p$ value by Fisher's exact test

** $p$ value by Wilcoxon test; DN vs. HRD, $p=0.0070$; HRD vs. GN, $p=0.0386$ 
Fig. 1 A Changes of annual numbers of dialysis initiation in Kesennuma city. B Changes of annual numbers of dialysis initiation based on the four causal renal diseases. A thick arrow indicates the occurrence of GEJE. $X$-axis is year and $Y$-axis is number. $D N$ diabetic nephropathy, $H R D$ hypertensive renal disease, $G N$ glomerulonephritis

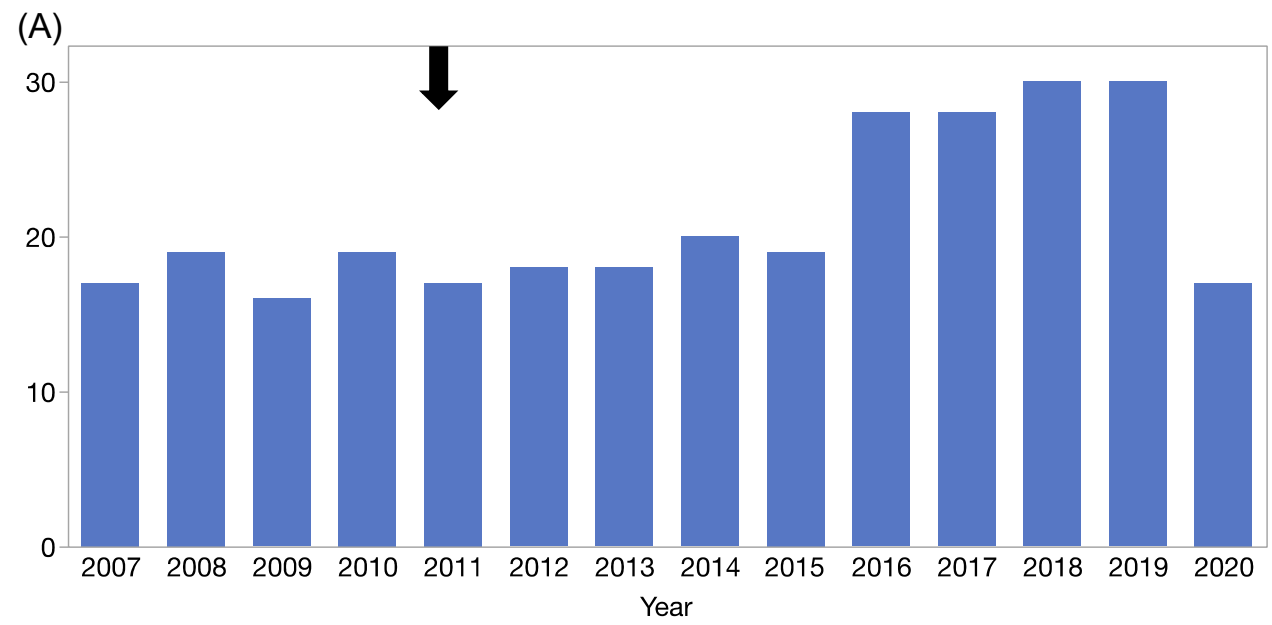

(B)

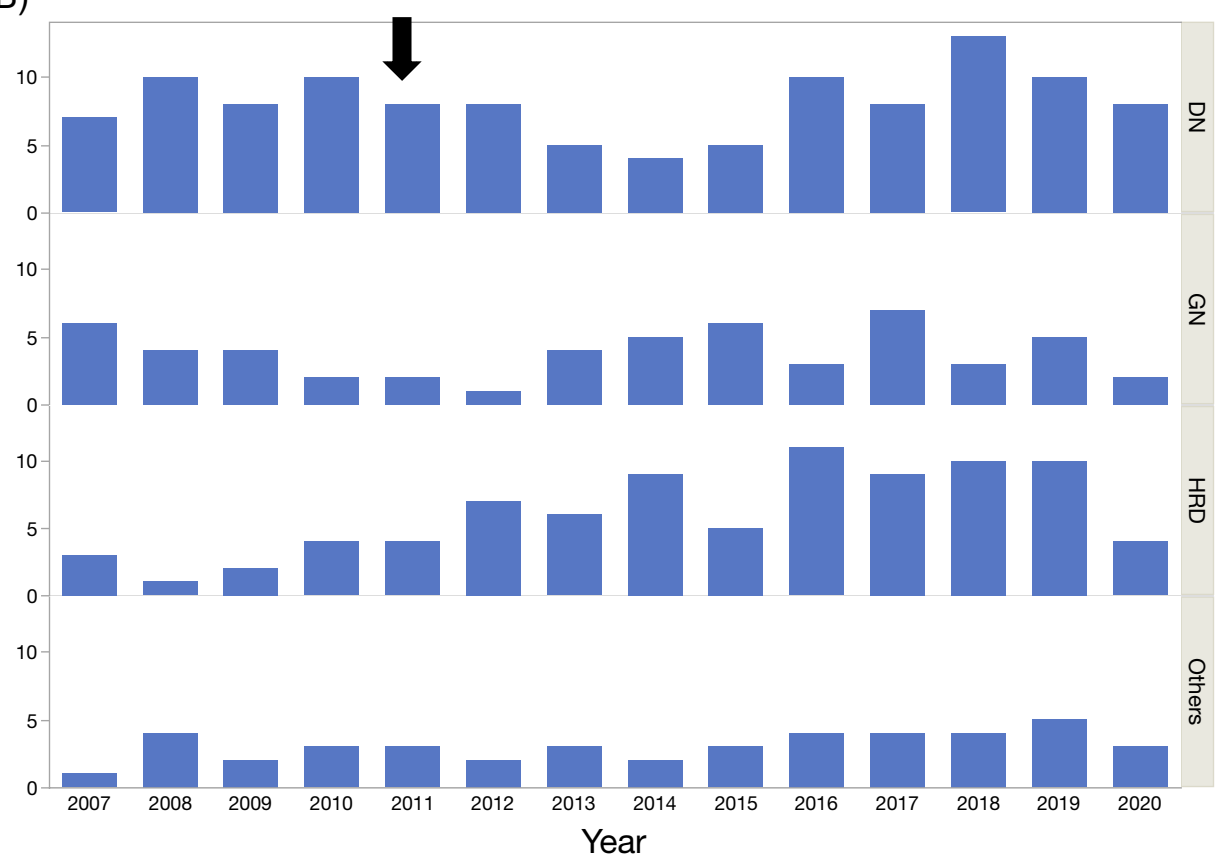

\section{Statistical analysis}

Comparisons of ratios between two or more groups were performed using Fisher's exact test and the Wilcoxon test. Statistical significance was set at a $p$ value of $<0.05$. Statistical analyses were performed using JMP ${ }^{\circledR}$ Pro 15.1.0 (SAS Campus Drive, NC, USA).

\section{Results}

Background data of the enrolled patients with dialysis initiation between 2007 and 2020 are summarized in Table 1. The total number of dialysis initiations was 296 (females, 91; age [mean \pm SD], $69.1 \pm 12.4$ years). Two patients underwent preemptive renal transplantation.

Renal diseases were: diabetic nephropathy $(n=114)$, hypertensive kidney diseases $(n=85)$, glomerulonephtits $(n=54)$, and others $(n=43)$. Fisher's exact test showed that there was no significant difference in the sex ratio for each causative disease $(p=0.5816)$. The Wilcoxon test showed a significant age difference $(p=0.0262)$. There was a significant difference in the number of cases in each disease group before and after the Great East Japan Earthquake $(p=0.0193)$. In particular, the ratio of hypertensive kidney diseases increased from $16.0 \%$ before the disaster to $33.5 \%$ thereafter. 
The sex and age of the patients according to the causative renal disease groups are shown in Table 2. Laboratory data upon dialysis initiation are reported in Supplementary Table 1.

Figure 1A shows the number of dialysis initiations before and after the Great East Japan Earthquake. The numberage atof dialysis initiation by four causal renal diseases is shown in Fig. 1B. Notably, the increase in dialysis initiation due to hypertension reached a plateau 5 years after the Great East Japan Earthquake, and returned to the before level 10 years later.

\section{Discussion}

This study shows a significant increase in the number of dialysis initiations 5 years after the Great East Japan Earthquake, which was sustained for a decade.

This increase was particularly associated with hypertensive renal diseases. Hypertension is a major, treatable cause of chronic kidney disease [7, 8]. As the African American Study of Kidney Disease and Hypertension Collaborative Research Group has reported, renal function continues to deteriorate even with well-controlled blood pressure and with low proteinuria in hypertensive patients [9]. Stress associated with disasters, especially if associated with a sense of loss, low income, and malnutrition, causes sustained activation of the sympathetic nervous system, which is a risk factor for the development and (poor) management of hypertension. Sodium intake may likewise be increased. This type of hypertension is known as disaster hypertension [10-12]. The increased frequency of cardiovascular events immediately after a natural disaster is attributed to the hyperactivation of the sympathetic nervous system, which increases blood pressure, and induces both endothelial dysfunction and hyperglycemia [1,2]. Inadequate blood pressure control is detrimental to the vascular system, enhancing the deterioration of renal function and arteriosclerosis [13, 14]. In line with this, hypertensive renal diseases needing dialysis increased after the Great East Japan Earthquake, as shown for the first time in our results [15]. While diabetic kidney disease remains a major cause of dialysis initiation, it did not share this increase.

A further reason for the increased incidence of hypertensive renal diseases after the Great East Japan Earthquake in Kesennuma City may be the aging of its citizens, while the younger population decreased, in particular after the earthquake. Overall, need for dialysis start increased $(p=0.0317)$ mainly due to hypertensive disorders (odds ratio post vs preearthquake: 2.52, $p=0.0037$ ) (Supplementary Table 2), and the increase was higher in the elderly (Supplementary Figures A, B, E, F).
This study has several limitations. First, data concerning the patients' blood pressure levels in the initial period after the Great East Japan Earthquake were not available. Nonetheless, we must consider that poor blood pressure was common in our in area for at least 1 year after the earthquake, which is in line with logistical constraints and psychological distress, both of which increase the risk of non-compliance [16]. Second, the role of the aging process is not clear. Third, this study was based on data from a single hospital. This is however the first study to suggest that a large natural disaster could lead to an increase in end stage kidney disease, and in particular due to hypertensive nephropathies.

This study also highlights the importance of conducting further studies on this topic, focusing on the long-term assessment of renal function and blood pressure after massive natural disasters.

Supplementary Information The online version contains supplementary material available at https://doi.org/10.1007/s40620-021-01140-9.

Acknowledgements We are particularly grateful to Dr. Kiyoshi Azumi, emeritus director of Kesennuna City Hospital, and Nrs. Michiyo Sato and Yuri Ogata, former chief nurses of the nephrology and dialysis units. We would also like to thank Editage (http://www.editage.com) for their English language editing services.

Author contributions MA planned and wrote the manuscript. ST and TI supported the design of the study. TA, AM and KI prepared the tables and figures. $\mathrm{KO}, \mathrm{HS}, \mathrm{HO}, \mathrm{KO}, \mathrm{MM}, \mathrm{KY}$ and TA provided intellectual input of critical importance to the work. All authors reviewed the manuscript.

Funding Individual funds from the Tohoku University Hospital discretionary research in 2019 were used in this study.

\section{Declarations}

Conflict of interest There is no conflict of interest.

Ethical approval This study was a retrospective observational study and was conducted in accordance with the Declaration of Helsinki.

Open Access This article is licensed under a Creative Commons Attribution 4.0 International License, which permits use, sharing, adaptation, distribution and reproduction in any medium or format, as long as you give appropriate credit to the original author(s) and the source, provide a link to the Creative Commons licence, and indicate if changes were made. The images or other third party material in this article are included in the article's Creative Commons licence, unless indicated otherwise in a credit line to the material. If material is not included in the article's Creative Commons licence and your intended use is not permitted by statutory regulation or exceeds the permitted use, you will need to obtain permission directly from the copyright holder. To view a copy of this licence, visit http://creativecommons.org/licenses/by/4.0/. 


\section{References}

1. Ogawa S, Ishiki M, Nako K, Okamura M, Senda M, Sakamoto T, Ito $S$ (2012) Effects of the Great East Japan Earthquake and huge tsunami on glycaemic control and blood pressure in patients with diabetes mellitus. BMJ Open 2:e000830

2. Kario K, McEwen BS, Pickering TG (2003) Disasters and the heart: a review of the effects of earthquake-induced stress on cardiovascular disease. Hypertens Res 26:355-367

3. Kloner RA (2019) Lessons learned about stress and the heart after major earthquakes. Am Heart J 215:20-26

4. Kario K, Matsuo T, Kobayashi H, Yamamoto K, Shimada K (1997) Earthquake-induced potentiation of acute risk factors in hypertensive elderly patients: possible triggering of cardiovascular events after a major earthquake. J Am Coll Cardiol 29:926-933

5. Abe M, Akaishi T, Ishii T (2021) Late increases in dialysis initiation after a massive disaster. Ther Apher Dial 25:125-126. https:// doi.org/10.1111/1744-9987.13498

6. He F, Xia X, Wu XF, Yu XQ, Huang FX (2013) Diabetic retinopathy in predicting diabetic nephropathy in patients with type 2 diabetes and renal disease: a meta-analysis. Diabetologia 56:457-466

7. Lee JY, Park JT, Joo YS, Lee C, Yun HR, Yoo TH, Kang SW, Choi KH, Ahn C, Oh KH, Sung S, Kim SW, Lee J, Han SH, KNOW-CKD (Korean Cohort Study for Outcomes in Patients with Chronic Kidney Disease) Investigators (2021) Association of blood pressure with the progression of CKD: findings from KNOW-CKD study. Am J Kidney Dis 78:236-245

8. Tozawa M, Iseki K, Iseki C, Kinjo K, Ikemiya Y, Takishita S (2003) Blood pressure predicts risk of developing end-stage renal disease in men and women. Hypertension 41:1341-1345

9. Appel LJ, Wright JT Jr, Greene T, Kusek JW, Lewis JB, Wang X, Lipkowitz MS, Norris KC, Bakris GL, Rahman M, Contreras G, Rostand SG, Kopple JD, Gabbai FB, Schulman GI, Gassman JJ, Charleston J, Agodoa LY, African American Study of Kidney Disease and Hypertension Collaborative Research Group (2008)
Long-term effects of renin-angiotensin system-blocking therapy and a low blood pressure goal on progression of hypertensive chronic kidney disease in African Americans. Arch Intern Med 168:832-839

10. Kario K (2012) Disaster hypertension - its characteristics, mechanism, and management -. Circ J 76:553-562. https://doi.org/10. 1253/circj.cj-11-1510

11. Sarki AM, Nduka CU, Stranges S, Kandala NB, Uthman OA (2015) Prevalence of hypertension in low- and middle-income countries: a systematic review and meta-analysis. Medicine 94:e1959. https://doi.org/10.1097/MD.0000000000001959

12. Mills KT, Bundy JD, Kelly TN, Reed JE, Kearney PM, Reynolds K, Chen J, He J (2016) Global disparities of hypertension prevalence and control: a systematic analysis of population-based studies from 90 countries. Circulation 134:441-450. https://doi. org/10.1161/CIRCULATIONAHA.115.018912

13. Zhang YP, Zuo XC, Huang ZJ, Kuang ZM, Lu MG, Duan DD et al (2013) The impact of blood pressure on kidney function in the elderly: a cross-sectional study. Kidney Blood Press Res 38:205-216

14. Ritz E (2010) Hypertension and kidney disease. Clin Nephrol 74(Suppl 1):S39-43

15. Ryan BJ, Franklin RC, Burkle FM, Smith EC, Aitken P, Leggat PA (2019) Determining key influences on patient ability to successfully manage noncommunicable disease after natural disaster. Prehosp Disaster Med 34:241-250

16. Nakaya N, Nakamura T, Tsuchiya N, Narita A, Tsuji I, Hozawa A, Tomita H (2017) Psychological distress and the risk of withdrawing from hypertension treatment after an earthquake disaster. Disaster Med Public Health Prep 11:179-182

Publisher's Note Springer Nature remains neutral with regard to jurisdictional claims in published maps and institutional affiliations. 